\title{
Local well-posedness of Musiela's SPDE with Lévy noise
}

\author{
Carlo Marinelli*
}

April 7, 2007. Revised April 21, 2008 and June 11, 2008.

\begin{abstract}
We determine sufficient conditions on the volatility coefficient of Musiela's stochastic partial differential equation driven by an infinite dimensional Lévy process so that it admits a unique local mild solution in spaces of functions whose first derivative is square integrable with respect to a weight.
\end{abstract}

2000 Mathematics Subject Classification: 60G51, 60H15, 91B28.

Keywords and phrases: HJM model, Musiela's stochastic PDE, stochastic PDEs with jumps, maximal inequalities.

\section{Introduction}

The aim of this paper is to find sufficient conditions on the volatility coefficient of Musiela's stochastic partial differential equation driven by Lévy noise such that it admits a unique mild (local) solution. In particular, denoting by $u(t, x)$ the forward rate prevailing at time $t$ with maturity $t+x, x \geq 0$, we shall consider the mild formulation of a stochastic PDE of the form

$$
d u(t, x)=\left[u_{x}(t, x)+f\left(t, x, u^{x}(t)\right)\right] d t+\langle\sigma(t, x, u(t, x)), d M(t)\rangle,
$$

where $u^{x}(t):=\{u(t, y): y \in[0, x]\}$ and $M$ is a locally square integrable martingale with independent increments taking values in a Hilbert space with inner product $\langle\cdot, \cdot\rangle$. Moreover, no-arbitrage conditions uniquely determine the functional form of $f$ in terms of $\sigma$ (see e.g. [5, 18, 24]). The properties of this SPDE in the case of $M$ being a Wiener process are rather well studied: let us just mention [11] for a self-contained treatment of existence of solutions, no-arbitrage conditions, and finite dimensional realizations, 13. for connections with Kolmogorov equations and option pricing, and 33. for the ergodic properties. A basic question to ask is clearly whether a solution exists to (1.1), and under what conditions on $\sigma$ and $f$. If $M$ is a Wiener process, one can draw on a large body of results (see e.g. [6]) to establish existence of solutions. In the general case of discontinuous $M$, the situation turns out to be more involved, not only because the no-arbitrage "constraint" on the drift term $f$ is relatively more complicated, but also (perhaps mainly) because the theory of stochastic PDEs driven

\footnotetext{
*Institut für Angewandte Mathematik, Universität Bonn, Wegelerstr. 6, D-53115 Bonn, Germany. URL: http://www. uni-bonn.de/ cm788
} 
by jump noise is not so well developed (see however, for instance, [14] and [27] for the variational approach, 22 for an analytic approach based on generalized Mehler semigroups, and [2, 28] for the "mild" approach).

The literature on HJM models driven by Lévy processes has considerably grown in the last few years: let us just cite, among others, [1, 7, 8, 9, and the seminal paper [5] (where general random measures are added to a finite dimensional Brownian motion as driving noises). All these papers essentially take as a starting point the existence of an HJM model with jumps, and concentrate on deriving no-arbitrage and completeness properties. Even though several of the papers just mentioned provide assumptions on the coefficients guaranteeing existence, an explicit study of sufficient conditions on the data of the corresponding Musiela stochastic PDE driven by Lévy noise guaranteeing (local or global) well-posedness seems to be missing, and it is the main motivation of the present paper. As mentioned above, we shall approach this problem studying the (Musiela-type) equation (1.1) using the semigroup approach to SPDEs (see e.g. [6]). Since this approach is mainly developed for the case of Brownian driving noise, we shall also need to prove some results on mild solutions of SPDEs driven by Lévy processes. Our main result is essentially local existence and uniqueness for (1.1) in an appropriate space of absolutely continuous functions, under suitable regularity and growth assumptions on $\sigma$ and on the Lévy measure of the noise $M$.

After this paper was finished, we found a preprint of Filipović and Tappe [12 where similar problems are investigated. However, these authors treat only the case of finitely many independent Lévy noises, and obtain solutions in an $L_{2}$ setting only. The techniques are also somewhat different, and ours seem to apply to more general equations as well (we refer in particular to the results of section (3). Moreover, a referee drew our attention to a preprint of Peszat and Zabczyk [29], which also deals with existence for Musiela's SPDE. As in [12, the Lévy process is finite dimensional and solutions are obtained in an $L_{2}$ setting. However, in contrast to [12] and the present paper, the state space is not contained in the space of continuous functions, hence irregular forward curves seem to be allowed.

The paper is organized as follows: in section 2 we introduce a general HJM model driven by an infinite dimensional Lévy noise via a Musiela-type SPDE, and we state two existence and uniqueness results. Section 3 contains general results on existence and uniqueness of mild solutions of SPDE with Lipschitz nonlinearities, which may be of independent interest. Important tools are maximal inequalities of Burkholder type, which are also proved in this section. Some more proofs are collected in section 4. and section 5 gives two examples covered by our results.

Let us conclude this introduction with some words about notation. By $a \lesssim b$ we mean that there exists a constant $N$ such that $a \leq N b$. To emphasize that the constant $N$ depends on a parameter $p$ we shall write $N(p)$ and $a \lesssim_{p} b$. Generic constants, which may change from line to line, are denoted by $N$. Given two separable Hilbert spaces $H, K$ we shall denote by $\mathcal{L}(K, H), \mathcal{L}_{0}(K, H), \mathcal{L}_{1}(K, H)$ and $\mathcal{L}_{2}(K, H)$ the space of linear, bounded linear, trace-class, and Hilbert-Schmidt operators, respectively, from $K$ to $H . \mathcal{L}_{1}^{+}$stands for the subset of $\mathcal{L}_{1}$ of positive operators. We shall write $\mathcal{L}_{1}(H)$ in place of $\mathcal{L}_{1}(H, H)$, and similarly for the other spaces. The Hilbert-Schmidt norm of $F \in \mathcal{L}_{2}$ is denoted by $|F|_{2}$. Given a self-adjoint operator 
$Q \in \mathcal{L}_{1}^{+}(K)$, we denote by $\mathcal{L}_{2}^{Q}(K, H)$ the set of all (possibly unbounded) operators $B: Q^{1 / 2} K \rightarrow H$ such that $B Q^{1 / 2} \in \mathcal{L}_{2}(K, H)$. The characteristic function of a set $A$ is denoted by $\chi_{A}$, and $\chi_{r}$ stands for the characteristic function of the set $B_{r}(H):=\{x \in H:|x| \leq r\}$, where $H$ is a Hilbert space. We denote by $|\cdot|_{p}$ the norm of the usual $L_{p}\left(\mathbb{R}_{+}\right)$spaces, $1 \leq p \leq \infty$. Given a continuously differentiable increasing function $\alpha: \mathbb{R}_{+} \rightarrow[1, \infty)$ such that $\alpha^{-1 / 3} \in L_{1}\left(\mathbb{R}_{+}\right)$, we define $L_{2, \alpha}^{n}:=L_{2, \alpha}^{n}\left(\mathbb{R}_{+}\right)$ as the space of distributions $\phi$ on $\mathbb{R}_{+}$such that

$$
|\phi|_{n, \alpha}^{2}:=\int_{0}^{\infty}\left|\phi^{(n)}(x)\right|^{2} \alpha(x) d x<\infty .
$$

We set $|\cdot|_{\alpha}:=|\cdot|_{0, \alpha}$. Moreover, the function $\alpha$ has to be considered fixed once and for all. For any Hilbert space $K$, we shall say that $\phi: \mathbb{R}_{+} \rightarrow K$ belongs to $L_{2, \alpha}^{n}\left(\mathbb{R}_{+}, K\right)$ if

$$
|\phi|_{n, \alpha, K}^{2}:=\int_{0}^{\infty}\left|\phi^{(n)}(x)\right|_{K}^{2} \alpha(x) d x<\infty
$$

and we shall write $|\cdot|_{\alpha, K}:=|\cdot|_{0, \alpha, K}$.

We shall use the symbol $D \varphi$ to denote the Fréchet derivative of a function $\varphi$.

\section{Setting and main results}

Let us begin stating precise assumptions on equation (1.1). We are given a real separable Hilbert space $K$ and a filtered probability space $(\Omega, \mathcal{F}, \mathbb{F}, \mathbb{P}), \mathbb{F}=\left(\mathcal{F}_{t}\right)_{t \in[0, T]}$, on which a right continuous square integrable martingale $M$ with values in $K$ is defined. We shall denote by $Q \in \mathcal{L}_{1}^{+}(K)$ the covariance operator of $M$, and by $\left(e_{k}\right)_{k \in \mathbb{N}}$ a (fixed) orthonormal basis of $K$. For any vector or function $\phi$ taking values in $K$ we set $\phi^{k}:=\left\langle\phi, e_{k}\right\rangle$. Moreover, we assume that $M$ has stationary independent increments and satisfies an exponential integrability condition. In particular, let $r>0$ be fixed, and assume that there exist positive constants $\delta>1$ and $C$ such that

$$
\mathbb{E} e^{|\langle\zeta, M(1)\rangle|}<C \quad \forall \zeta \in B_{\delta r}(K) .
$$

Let us also define the function

$$
\psi(\zeta):=\log \mathbb{E} e^{\langle\zeta, M(1)\rangle}, \quad \zeta \in B_{\delta r}(K) .
$$

The function $\psi$ admits the representation

$$
\psi(\zeta)=-\frac{1}{2}\langle R \zeta, \zeta\rangle+\int_{K}\left(e^{\langle\zeta, x\rangle}-1-\langle\zeta, x\rangle\right) m(d x),
$$

where $R$ and $m$ denote the covariance operator of the Brownian component and the Lévy measure of $M$, respectively. Given a real number $p \geq 2$, we shall write $M \in \mathcal{M}_{p}$ if $M(0)=0$ and

$$
\int_{K}|x|^{q} m(d x)<\infty \quad \forall q \in[2, p] .
$$

The (random) function $\sigma:[0, T] \times \mathbb{R}_{+} \times \mathbb{R} \rightarrow K$ is measurable, predictable, and satisfies

$$
\int_{0}^{x} \sigma(t, y, u(y)) d y \in B_{r}(K) \quad \forall(t, x, u(\cdot)) \in[0, T] \times \mathbb{R}_{+} \times L_{2, \alpha}^{1}
$$


almost surely.

From now on we shall assume that the measure $\mathbb{P}$ is such that discounted zerocoupon bond prices are local martingales. Let us recall that denoting by $B(t, x)$ the price at time $t \geq 0$ of a zero-coupon bond paying one at time $t+x$ (with $x \geq 0$ ), the following identity holds

$$
B(t, x)=\exp \left(-\int_{0}^{x} u(t, y) d y\right) .
$$

Defining the mapping

$$
g(\nu)(t, x)=\left\langle\nu(t, x), D \psi\left(-\int_{0}^{x} \nu(t, y) d y\right)\right\rangle
$$

on functions $\nu:[0, T] \times \mathbb{R}_{+} \rightarrow K$ such that $\int_{0}^{x} \nu(t, y) d y \in B_{r}(K)$ for all $(t, x) \in$ $[0, T] \times \mathbb{R}_{+}$, the following functional relationship holds between $f$ and $\sigma$ :

$$
f(t, x)=g(\sigma)(t, x) .
$$

Note that in (2.3) the Fréchet derivative $D \psi$ is well defined thanks to proposition 3.1 below.

Clearly, writing $\sigma(t, x) \equiv \sigma(t, x, u(x))$ for some function $u$, the above expression for $f$ is equivalent to

$$
f(t, x, u(\cdot))=\left\langle\sigma(t, x, u(x)), D \psi\left(-\int_{0}^{x} \sigma(t, y, u(y)) d y\right)\right\rangle .
$$

In particular note that, in view of the previous expression, in (1.1) we cannot write, in general, $f(t, x, u(t, x))$, but we must write instead $f\left(t, x, u^{x}(t)\right)$. More regularity assumptions on $\sigma$, hence on $f$, will be stated or proved as needed. Finally, equation (1.1) is supplemented with an initial condition $u(0, x)=u_{0}(x)$, with $u_{0}$ an $L_{2, \alpha}^{1}$-valued random variable measurable with respect to $\mathcal{F}_{0}$.

We shall rewrite the SPDE (1.1) as an abstract stochastic differential equation in the space $H=L_{2, \alpha}^{1}$, which becomes a separable Hilbert space when endowed with the inner product

$$
\langle\phi, \psi\rangle_{H}:=\int_{\mathbb{R}_{+}} \phi^{\prime}(x) \psi^{\prime}(x) \alpha(x) d x+\phi(0) \psi(0) .
$$

We shall often use the following properties of the space $H$, for a proof of which we refer to [11, sect. 5.1]. In particular, for a function $\phi \in H$, we have

$$
\begin{aligned}
|\phi|_{\infty} & \lesssim|\phi|_{H}, \\
|(\phi-\phi(\infty))|_{1} & \lesssim|\phi|_{H}, \\
\left|(\phi-\phi(\infty))^{2}\right|_{\alpha} & \lesssim|\phi|_{H}^{2},
\end{aligned}
$$

where the constants depend only on $\alpha$. This state space has apparently been first used by Filipović [11]. Other authors have considered related SPDEs in different function spaces, e.g. in weighted $L_{2}$ spaces, weighted Sobolev spaces, or fractional Sobolev spaces (see [13], 34], 10] respectively). 
Let us define on $H$ the operator $A: \phi \mapsto \phi^{\prime}$, with domain $D(A)=L_{2, \alpha}^{1} \cap L_{2, \alpha}^{2}$, which generates the semigroup of right shifts $e^{t A} \phi(x):=\phi(x+t), t \geq 0$. We can formally rewrite Musiela's SPDE (1.1) in the following abstract form:

$$
d u(t)=[A u(t)+f(t, u(t))] d t+B(t, u(t)) d M(t), \quad u(0)=u_{0},
$$

where $f:[0, T] \times H \rightarrow H$ is defined by $x \mapsto g(\sigma)(t, x)$ (with a slight but harmless abuse of notation), and $B:[0, T] \times H \rightarrow \mathcal{L}(K, H)$ is defined by $[B(t, u) \phi](x)=$ $\langle\sigma(t, x, u(x)), \phi\rangle_{K}, \phi \in K$. This rewriting is, for the time being, only formal, because one needs to prove that the images of $f$ and $B$ are actually contained in $H$ and $\mathcal{L}_{Q}^{2}(K, H)$, which is not true without further assumptions. For now, however, we content ourselves with the formal equation (2.7), noting that it can be made rigorous under conditions that will be given in theorems 2.3 and 2.6 below.

The concept of solution we shall work with and the space where mild solutions are sought are defined next.

Definition 2.1. A predictable process $u:[0, T] \times \Omega \rightarrow H$ is a mild solution of (2.7) if it satisfies

$$
u(t)=e^{t A} u_{0}+\int_{0}^{t} e^{(t-s) A} f(s, u(s)) d s+\int_{0}^{t} e^{(t-s) A} B(s, u(s)) d M(s)
$$

$\mathbb{P}$-a.s. for almost all $t \in[0, T]$.

Definition 2.2. We shall denote by $\mathcal{H}_{p}(T), p \geq 2$, the space of all predictable processes $u:[0, T] \rightarrow H$ such that

$$
|[u]|_{p}^{p}:=\sup _{t \in[0, T]} \mathbb{E}|u(t)|^{p}<\infty .
$$

We have the following local well-posedness result in $\mathcal{H}_{2}(T)$.

Theorem 2.3. Assume that

(i) $\sigma^{k} \in C^{0,1,2}\left([0, T] \times \mathbb{R}_{+} \times \mathbb{R}\right)$ for all $k \in \mathbb{N}$;

(ii) there exists $\beta \in L_{2, \alpha}\left(\mathbb{R}_{+}, \ell_{2}\right)$ such that, for all $k \in \mathbb{N}$,

$$
\left|\sigma_{x}^{k}(t, x, u)-\sigma_{x}^{k}(t, x, v)\right| \leq \beta^{k}(x)|u-v|
$$

for almost all (a.a.) $(t, x) \in[0, T] \times \mathbb{R}_{+}, \mathbb{P}$-a.s.;

(iii) there exists $\gamma=\left(\gamma^{k}\right)_{k \in \mathbb{N}} \in \ell_{2}$ such that

$$
\left|\sigma_{u}^{k}(t, x, u)\right|+\left|\sigma_{u u}^{k}(t, x, u)\right| \leq \gamma^{k}
$$

for a.a. $(t, x, u) \in[0, T] \times \mathbb{R}_{+} \times \mathbb{R}, \mathbb{P}$-a.s.;

(iv) there exists a constant $C$ such that $|\sigma(t, \cdot, 0)|_{1, \alpha, K}<C$ for a.a. $t \in[0, T], \mathbb{P}$-a.s.;

(v) $\mathbb{E}\left|u_{0}\right|^{2}<\infty$. 
Then (1.1) has a unique local mild solution in $\mathcal{H}_{2}(T)$. Furthermore, the solution depends Lipschitz continuously on the initial datum.

Remark 2.4. In (2.9) one could replace the second term on the left hand side with $\left[\sigma_{u}^{k}(t, x, u)\right]_{\text {Lip }}$, where $[\cdot]_{\text {Lip }}$ stands for the Lipschitz norm with respect to the third variable. In other words, the same proof goes through if one replaces the assumption on boundedness of the second derivative of $\sigma^{k}$ with respect to $u$ with an assumption of Lipschitz continuity of $\sigma_{u}^{k}$ with uniform Lipschitz constant.

The proof of this theorem (which is given in section 4) relies on a more general result on existence and uniqueness for stochastic evolution equations with Lipschitz nonlinearities (see theorem 3.5 below), combined with regularity results for $f$.

The above theorem, as well as theorem 3.5 below, are stated and proved for their simplicity, even though a more refined result holds true. In fact, one can look for mild solutions of (2.7) in smaller spaces that are the infinite dimensional version of the spaces usually considered in the theory of finite dimensional SDEs (see e.g. [31]).

Definition 2.5. We shall denote by $\mathbb{H}_{p}(T), p \geq 2$, the space of all predictable processes $u:[0, T] \rightarrow H$ such that

$$
\|u\|_{p}^{p}:=\mathbb{E} \sup _{t \in[0, T]}|u(t)|^{p}<\infty .
$$

The following local well-posedness result in $\mathbb{H}_{p}(T)$ is the main result of this paper.

Theorem 2.6. Let $p \geq 2$ and assume that hypotheses (i)-(iv) of theorem 2.3 hold. Moreover, assume that $\mathbb{E}\left|u_{0}\right|^{p}<\infty$ and $M \in \mathcal{M}_{p}$. Then (1.1) has a unique local mild solution in $\mathbb{H}_{p}(T)$. Furthermore, the solution depends Lipschitz continuously on the initial datum.

\section{Auxiliary results}

Let us start proving a regularity result for the function $\psi$ defined in (2.2) above, under the assumption (2.1).

Proposition 3.1. One has $\psi \in C_{b}^{2}\left(B_{r}(K)\right)$ and $D^{2} \psi$ is Lipschitz on $B_{r}(K)$.

Proof. We claim that the Gâteaux derivative of $\psi$ at a point $\zeta \in B_{r}(K)$ is given by

$$
D_{G} \psi(\zeta): \phi \mapsto\langle R \zeta, \phi\rangle+\int_{K}\langle x, \phi\rangle\left(e^{\langle\zeta, x\rangle}-1\right) m(d x)
$$

In fact, for any $\zeta \in B_{r}(K)$ and $\xi \in K$, we have

$$
\begin{aligned}
\lim _{\varepsilon \rightarrow 0} \frac{\psi(\zeta+\varepsilon \xi)-\psi(\zeta)}{\varepsilon} & =\langle R \zeta, \xi\rangle+\lim _{\varepsilon \rightarrow 0} \int_{K}\left(e^{\langle\zeta, x\rangle} \frac{e^{\varepsilon\langle\xi, x\rangle}-1}{\varepsilon}+\langle\xi, x\rangle\right) m(d x) \\
& =\langle R \zeta, \xi\rangle+\int_{K}\langle\xi, x\rangle\left(e^{\langle\zeta, x\rangle}-1\right) m(d x) .
\end{aligned}
$$


The passage to the limit under the integral sign in the above expression is possible thanks to the dominated convergence theorem. In fact, by the mean value theorem, one has

$$
e^{\langle\zeta, x\rangle} \frac{e^{\varepsilon\langle\xi, x\rangle}-1}{\varepsilon}=e^{\langle\zeta, x\rangle} e^{\varepsilon \eta\langle\xi, x\rangle}\langle\xi, x\rangle
$$

for some $\eta \in(0,1)$, hence

$$
\left|e^{\langle\zeta, x\rangle} \frac{e^{\varepsilon\langle\xi, x\rangle}-1}{\varepsilon}\right| \leq|\xi||x| e^{|\langle\zeta, x\rangle|} e^{\varepsilon|\langle\xi, x\rangle|},
$$

and, setting $\delta^{\prime}=\delta /(\delta-1)$ and appealing to Hölder's inequality,

$$
\begin{aligned}
& \int_{K}|x| e^{|\langle\zeta, x\rangle|} e^{\varepsilon|\langle\xi, x\rangle|} m(d x) \\
& \quad \leq\left(\int_{K} e^{|\langle\delta \zeta, x\rangle|} m(d x)\right)^{\frac{1}{\delta}}\left(\int_{K}|x|^{2 \delta^{\prime}} m(d x)\right)^{\frac{1}{2 \delta^{\prime}}}\left(\int_{K} e^{2 \varepsilon \delta^{\prime}|\langle\xi, x\rangle|} m(d x)\right)^{\frac{1}{2 \delta^{\prime}}} \\
& \quad<\infty,
\end{aligned}
$$

for all $\varepsilon<\left(2 \delta^{\prime}|\xi|\right)^{-1} \delta r$.

Let us now show that $D_{G} \psi$ is continuous from $K$ to $\mathcal{L}(K, \mathbb{R})$. Let $\zeta_{n} \rightarrow \zeta$ as $n \rightarrow \infty$, $h \in K$. Then

$$
\begin{aligned}
\sup _{|h| \leq 1}\left|D_{G} \psi\left(\zeta_{n}\right) h-D_{G} \psi(\zeta) h\right| & \leq \sup _{|h| \leq 1} \int_{K}|\langle x, h\rangle|\left|e^{\left\langle\zeta_{n}, x\right\rangle}-e^{\langle\zeta, x\rangle}\right| m(d x) \\
& \leq \int_{K}|x|\left|e^{\left\langle\zeta_{n}, x\right\rangle}-e^{\langle\zeta, x\rangle}\right| m(d x) \\
& \rightarrow 0
\end{aligned}
$$

as $n \rightarrow \infty$ by the dominated convergence theorem, with computations completely analogous to the above ones. Since $D_{G} \psi$ is continuous, then $D \psi=D_{G} \psi$ by a wellknown criterion for Fréchet differentiability (see e.g. [3, thm. 1.9]).

Proceeding in a completely similar way one can prove that $\psi$ is twice Fréchet differentiable in $B_{r}(K)$, with

$$
D^{2} \psi(\zeta):(\phi, \eta) \mapsto \int_{K}\langle x, \phi\rangle\langle x, \eta\rangle e^{\langle x, \zeta\rangle} m(d x) .
$$

The boundedness of $D \psi$ on $B_{r}(K)$ can again be proved by applying Hölder's inequality to (3.1).

In a completely analogous way one can prove that $D^{2} \psi$ as well as $D^{3} \psi$ are bounded on $B_{r}(K)$, in particular $D^{2} \psi$ is Lipschitz.

Remark 3.2. Simpler but less general assumptions than (2.1) may be given. In fact, (2.1) is clearly satisfied if $\int e^{\delta r|x|} m(d x)<\infty$. Another sufficient condition for (2.1) is that the map $\varphi: \zeta \mapsto \int_{K} e^{|\langle x, \zeta\rangle|} m(d x)$ is continuous with respect to the weak topology of $K$. In fact, by Banach-Alaoglu's theorem, the ball $B_{\delta r}$ is weakly compact in $K$, hence the image of $B_{\delta r}$ under $\varphi$ is compact in the real line and $\varphi$ attains a (finite) maximum at some point $\zeta_{0} \in B_{\delta r}$. 
Let us denote by $[M]$ and $\langle M\rangle$ the quadratic variation and the Meyer process of $M$, respectively. We shall need the following elementary lemma, whose proof is included for completeness.

Lemma 3.3. There exists a (deterministic) operator $Q \in \mathcal{L}_{1}^{+}(K)$ such that $\langle M\rangle(t)=$ $t \operatorname{Tr}(Q)$ for all $t \geq 0$.

Proof. Let $Q$ be the correlation operator of $M(1)$, i.e. the operator defined by

$$
\langle Q x, y\rangle=\mathbb{E}\langle M(1), x\rangle\langle M(1), y\rangle, \quad x, y \in K .
$$

Recalling that $\mathbb{E}|M(1)|^{2}<\infty$, it is immediate to prove that $Q \in \mathcal{L}_{1}^{+}(K)$. Since $M$ has homogeneous independent increments, we also have

$$
\mathbb{E}\left[|M(t)|^{2}-|M(s)|^{2} \mid \mathcal{F}_{s}\right]=\mathbb{E}\left[|M(t)-M(s)|^{2}\right]=(t-s) \operatorname{Tr}(Q),
$$

where for the second equality we have used the facts that $M(t)-M(s)=M(t-s)$ in distribution, $\mathbb{E}|M(t)|^{2}=t \mathbb{E}|M(1)|^{2}$, and $\mathbb{E}|M(1)|^{2}=\operatorname{Tr}(Q)$, as follows by definition of $Q$.

Let $E$ be a real separable Hilbert spaces, and $A: D(A) \subset E \rightarrow E$ the generator of a strongly continuous semigroup $e^{t A}$ on $E$. We will extensively use the following isometric formula due to Métivier and Pistone [26]: let $Q$ be the correlation operator of $M$, and $F:[0, T] \rightarrow \mathcal{L}_{Q}^{2}(K, E)$ a predictable process such that

$$
\mathbb{E} \int_{0}^{T}|F(s)|_{Q}^{2} d s<\infty
$$

Then we have

$$
\mathbb{E}\left|\int_{0}^{t} F(s) d M(s)\right|^{2}=\mathbb{E} \int_{0}^{t}|F(s)|_{Q}^{2} d s .
$$

Remark 3.4. In fact the isometric formula of [26] states that for every square integrable martingale $M$ and $F$ as above such that

$$
\mathbb{E} \int_{0}^{t}|F(s)|_{Q_{M}(s)}^{2} d\langle M\rangle(s)<\infty \quad \forall t \leq T,
$$

one has

$$
\mathbb{E}\left|\int_{0}^{t} F(s) d M(s)\right|^{2}=\mathbb{E} \int_{0}^{t}|F(s)|_{Q_{M}(s)}^{2} d\langle M\rangle(s),
$$

where $Q_{M}:[0, T] \rightarrow \mathcal{L}_{1}^{+}(K)$ is a predictable process with $\operatorname{Tr}\left(Q_{M}(s)\right)=1$ a.s., for all $s \leq T$. If $M$ has independent increments, then one also has $Q_{M}(t)=Q / \operatorname{Tr}(Q)$, hence (3.2) follows immediately by (3.3) and lemma 3.3 .

In this section we establish existence and uniqueness of mild solutions of equations of the form (2.7) on a general Hilbert space $E$, assuming only $A$ generates a strongly continuous semigroup (resp. strongly continuous contraction semigroup) and the nonlinear terms $f$ and $B$ are maps satisfying a Lipschitz assumptions.

In the following theorem we shall denote by $\Psi: L_{2}(\Omega, \mathcal{F}, \mathbb{P} ; H) \rightarrow \mathcal{H}_{2}(T)$ the solution map of (2.7), that is, $\Psi(\eta)$ stands for the solution of (2.7) with initial condition $\eta$. To simplify notation, we shall set $\mathcal{H}_{p}=L_{p}(\Omega, \mathcal{F}, \mathbb{P} ; H)$ for $p \geq 2$. 
Theorem 3.5. Assume that $\mathbb{E}\left|u_{0}\right|^{2}<\infty, e^{s A} B(t, x) \in \mathcal{L}_{2}^{Q}(K, E)$ for all $(t, x) \in$ $[0, T] \times E$, and there exists $h \in L_{2, l o c}(\mathbb{R})$ such that, for all $s, t \in[0, T]$,

$$
\begin{gathered}
\left|e^{s A} f(t, x)\right|+\left|e^{s A} B(t, x)\right|_{Q} \leq h(s)(1+|x|), \\
\left|e^{s A}(f(t, x)-f(t, y))\right|+\left|e^{s A}(B(t, x)-B(t, y))\right|_{Q} \leq h(s)|x-y| .
\end{gathered}
$$

for all $x, y \in E, \mathbb{P}$-a.s. Then equation (2.7) admits a unique mild solution in $\mathcal{H}_{2}(T)$. Moreover, there exists a constant $N$, independent of $u_{0}, v_{0}$, such that

$$
\left|\left[\Psi\left(u_{0}\right)-\Psi\left(v_{0}\right)\right]\right|_{2} \leq N\left|u_{0}-v_{0}\right|_{\mathcal{H}_{2}}
$$

for all $u_{0}, v_{0} \in \mathcal{H}_{2}$.

Proof. The proof follows the same reasoning used for equations with Wiener noise. Some redundant details are therefore omitted. We shall prove that the mapping $\mathfrak{F}: \mathcal{H}_{2}(T) \rightarrow \mathcal{H}_{2}(T)$ defined by

$$
\mathfrak{F} u(t)=e^{t A} u_{0}+\int_{0}^{t} e^{(t-s) A} f(s, u(s)) d s+\int_{0}^{t} e^{(t-s) A} B(s, u(s)) d M(s)
$$

is well defined and is a contraction, after which the result will follow easily by Banach's fixed point theorem. Let us first prove that the image of $\mathfrak{F}$ is in fact contained in $\mathcal{H}_{2}(T)$ : to this purpose, we have to show that, for any $u \in \mathcal{H}_{2}(T)$, $\mathfrak{F} u$ admits a predictable modification and that $|[\mathfrak{F} u]|_{2}<\infty$. Predictability of $\mathfrak{F} u$ follows by the mean-square continuity of the stochastic convolution term in (3.7): in fact, setting $M_{A}(t)=\int_{0}^{t} e^{(t-s) A} B(s, u(s)) d M(s)$, a simple calculation shows that, for $0 \leq s \leq t \leq$ $T$,

$$
\begin{aligned}
\mathbb{E}\left|M_{A}(t)-M_{A}(s)\right|^{2} \lesssim & \mathbb{E}\left|\int_{0}^{s}\left(e^{(t-r) A}-e^{(s-r) A}\right) B(r, u(r)) d M(r)\right|^{2} \\
& +\mathbb{E}\left|\int_{s}^{t} e^{(t-r) A} B(r, u(r)) d M(r)\right|^{2} \\
\leq & \mathbb{E} \int_{0}^{s}\left|e^{(t-r) A}-e^{(s-r) A}\right|^{2}|B(r, u(r))|_{Q}^{2} d r \\
& +\mathbb{E} \int_{s}^{t}\left|e^{(t-r) A}\right|^{2}|B(r, u(r))|_{Q}^{2} d r,
\end{aligned}
$$

where the second inequality follows by the isometric formula for stochastic integrals. Therefore $\mathbb{E}\left|M_{A}(t)-M_{A}(s)\right|^{2} \rightarrow 0$ as $s \rightarrow t$.

Moreover, we have

$$
\begin{aligned}
|[\mathfrak{F} u]|_{2}^{2} \lesssim & \sup _{t \leq T} \mathbb{E}\left|e^{t A} u_{0}\right|^{2}+\sup _{t \leq T} \mathbb{E}\left|\int_{0}^{t} e^{(t-s) A} f(s, u(s)) d s\right|^{2} \\
& +\sup _{t \leq T} \mathbb{E}\left|\int_{0}^{t} e^{(t-s) A} B(s, u(s)) d M(s)\right|^{2}
\end{aligned}
$$

and $\sup _{t \leq T} \mathbb{E}\left|e^{t A} u_{0}\right|^{2} \leq \sup _{t \leq T}\left|e^{t A}\right|^{2} \mathbb{E}\left|u_{0}\right|^{2}<\infty$. Cauchy-Schwarz' inequality and (3.4) yield

$$
\mathbb{E}\left|\int_{0}^{t} e^{(t-s) A} f(s, u(s)) d s\right|^{2} \leq 2 T \mathbb{E} \int_{0}^{t} h^{2}(t-s)\left(1+|u(s)|^{2}\right) d s,
$$


hence

$$
\sup _{t \leq T} \mathbb{E}\left|\int_{0}^{t} e^{(t-s) A} f(s, u(s)) d s\right|^{2} \leq N \sup _{t \leq T} \mathbb{E}\left(1+|u(t)|^{2}\right)<\infty,
$$

where $N=2 T \int_{0}^{T} h^{2}(s) d s$.

Similarly, using the isometric formula (3.2), lemma 3.3 and (3.4), we have

$$
\begin{aligned}
\mathbb{E}\left|\int_{0}^{t} e^{(t-s) A} B(s, u(s)) d M(s)\right|^{2} & =\mathbb{E} \int_{0}^{t}\left|e^{(t-s) A} B(s, u(s)) Q^{1 / 2}\right|_{2}^{2} d s \\
& \leq \mathbb{E} \int_{0}^{t} h(t-s)^{2}(1+|u(s)|)^{2} d s .
\end{aligned}
$$

Then

$$
\sup _{t \leq T} \mathbb{E}\left|\int_{0}^{t} e^{(t-s) A} B(s, u(s)) d M(s)\right|^{2} \leq N \sup _{t \leq T} \mathbb{E}\left(1+|u(t)|^{2}\right)
$$

with $N=2 \int_{0}^{T} h^{2}(s) d s$. Therefore we have

$$
|[\mathfrak{F} u]|_{2}^{2}=\sup _{t \leq T} \mathbb{E}|\mathfrak{F} u(t)|^{2} \lesssim 1+\sup _{t \leq T} \mathbb{E}|u(t)|^{2}<\infty,
$$

which proves that $\mathfrak{F} u \in \mathcal{H}_{2}(T)$.

Completely analogous calculations involving (3.5) instead of (3.4) show that

$$
|[\mathfrak{F} u-\mathfrak{F} v]|_{2}^{2} \leq N|[u-v]|_{2}^{2},
$$

with

$$
N=N(T, h)=2(T+1) \int_{0}^{T} h^{2}(s) d s .
$$

Since $h \in L_{2, l o c}(\mathbb{R})$, one can find $T_{0}>0$ so that $N\left(T_{0}, h\right)<1$, thus one obtains, by Banach's fixed point theorem, existence and uniqueness of a mild solution to (2.7) on the time interval $\left[0, T_{0}\right]$. Then one can proceed with classical extension arguments, proving that a global solution on the time interval $[0, T]$ exists and is unique.

In order to prove (3.6), it is convenient to regard the map $\mathfrak{F}$ as a function from $\mathcal{H}_{2} \times \mathcal{H}_{2}(T)$ to $\mathcal{H}_{2}(T)$. Let us assume, without loss of generality, that $T$ is such that $\left|\left[\mathfrak{F}\left(u_{0}, u\right)-\mathfrak{F}\left(u_{0}, v\right)\right]\right|_{2} \leq N_{1}|[u-v]|_{2}$ with $N_{1}<1$. Then the above fixed-point argument implies that the solution map $\Psi: \mathcal{H}_{2} \rightarrow \mathcal{H}_{2}(T)$ is such that $\mathfrak{F}\left(u_{0}, \Psi\left(u_{0}\right)\right)=$ $\Psi\left(u_{0}\right)$. Moreover, by definition of $\mathfrak{F}$, we have that

$$
\left|\left[\mathfrak{F}\left(u_{0}, w\right)-\mathfrak{F}\left(v_{0}, w\right)\right]\right|_{2} \leq N_{2}\left|u_{0}-v_{0}\right|_{\mathcal{H}} \quad \forall w \in \mathcal{H}_{2}(T),
$$

with $N_{2}=\left(\sup _{t \leq T}\left|e^{t A}\right|\right)^{1 / 2}$. Therefore we can write

$$
\begin{aligned}
\left|\left[\Psi\left(u_{0}\right)-\Psi\left(v_{0}\right)\right]\right|_{2}= & \left|\left[\mathfrak{F}\left(u_{0}, \Psi\left(u_{0}\right)\right)-\mathfrak{F}\left(u_{0}, \Psi\left(u_{0}\right)\right)\right]\right|_{2} \\
\leq & \left|\left[\mathfrak{F}\left(u_{0}, \Psi\left(u_{0}\right)\right)-\mathfrak{F}\left(u_{0}, \Psi\left(v_{0}\right)\right)\right]\right|_{2} \\
& +\left|\left[\mathfrak{F}\left(u_{0}, \Psi\left(v_{0}\right)\right)-\mathfrak{F}\left(v_{0}, \Psi\left(v_{0}\right)\right)\right]\right|_{2} \\
\leq & N_{1}\left|\left[\Psi\left(u_{0}\right)-\Psi\left(v_{0}\right)\right]\right|_{2}+N_{2}\left|u_{0}-v_{0}\right|_{\mathcal{H}_{2}},
\end{aligned}
$$

hence $\left|\left[\Psi\left(u_{0}\right)-\Psi\left(v_{0}\right)\right]\right|_{2} \leq\left(1-N_{1}\right)^{-1} N_{2}\left|u_{0}-v_{0}\right|_{\mathcal{H}_{2}}$. 
Remark 3.6. In order to obtain existence and uniqueness in $\mathcal{H}_{p}(T), p>2$, one would need an estimate for a term of the type

$$
\sup _{t \leq T} \mathbb{E}\left|\int_{0}^{t} e^{(t-s) A} F(s) d M(s)\right|^{p}
$$

with $F$ a predictable function, whereas for the proof in $\mathcal{H}_{2}(T)$ the isometric property of the stochastic integral is enough. An estimate of Burkholder type in $\mathcal{H}_{p}(T)$, $p>2$, for stochastic convolutions with respect to compensated Poisson measures is announced in [19]. The maximal inequality (3.14) seems to be simpler and more natural, even though it holds only for pseudo-contraction semigroups $e^{t A}$.

The main result of this section is the following theorem, where the solution map $\Psi$ is now defined from $\mathcal{H}_{p}=L_{p}(\Omega, \mathcal{F}, \mathbb{P} ; H)$ to $\mathbb{H}_{p}(T)$.

Theorem 3.7. Let $p \geq 2$. Assume that $e^{t A}$ is a contraction semigroup, $\mathbb{E}\left|u_{0}\right|^{p}<\infty$, $M \in \mathcal{M}_{p}$, and there exists $h \in L_{p, l o c}(\mathbb{R})$ such that

$$
\begin{gathered}
\left|e^{s A} f(t, x)\right|+|B(s, x)| \leq h(s)(1+|x|), \\
\left|e^{s A}(f(t, x)-f(t, y))\right|+|B(s, x)-B(s, y)| \leq h(s)|x-y| .
\end{gathered}
$$

for all $x, y \in E$. Then equation 2.7) admits a unique mild solution in $\mathbb{H}_{p}(T)$. Moreover, there exists a constant $N$, independent of $u_{0}, v_{0}$, such that

$$
\left\|\Psi\left(u_{0}\right)-\Psi\left(v_{0}\right)\right\|_{p} \leq N\left|u_{0}-v_{0}\right|_{\mathcal{H}_{p}}
$$

for all $u_{0}, v_{0} \in \mathcal{H}_{p}$.

Note that in order to obtain a solution in $\mathbb{H}_{p}(T)$, which is a subset of $\mathcal{H}_{p}(T)$, one has to assume that $A$ generates a contraction semigroup, which was not assumed in theorem 3.5 .

In order to prove theorem 3.7 we need to establish a maximal inequality of Burkholder type for stochastic convolutions, which may be of independent interest. For related estimates, which could probably be used in this context as well, see also [15, 17, 20, 21].

Let us first recall the Burkholder inequality for Hilbert space valued martingales, see e.g. [25, §6.E.3].

Proposition 3.8. Let $X$ be a $K$-valued local martingale with $X(0)=0$. For every $p \in[2, \infty)$ there exists a constant $C(p)$ depending on $p$ only such that

$$
\mathbb{E} \sup _{t \leq T}|X(t)|^{p} \leq C(p) \mathbb{E}[X](T)^{p / 2} .
$$

For $F$ predictable and locally bounded, setting $X(t)=\int_{0}^{t} F(s) d M(s)$, (3.11) implies

$$
\mathbb{E} \sup _{t \leq T}\left|\int_{0}^{t} F(s) d M(s)\right|^{p} \leq C(p) \mathbb{E}\left(\int_{0}^{T}|F(s)|^{2} d[M](s)\right)^{p / 2} .
$$

This estimate allows to obtain a maximal inequality of Bichteler-Jacod type in Hilbert space, following very closely the proof of lemma 4.1 in [30]. That is, we have the following lemma, of which we include a proof for the reader's convenience. 
Lemma 3.9. Let $p \geq 2$. Let $Y$ be a martingale Lévy process with $Y(0)=0$ and $\int_{K}|x|^{q} m(d x)<\infty$ for all $q \in[2, p]$, where $m$ is the Lévy measure of $Y$. Let $F$ be a predictable locally bounded process such that $\mathbb{E} \int_{0}^{T}|F(s)|^{p} d s<\infty$. Then there exists a constant $N$ which depends only on $p$ and $T$ such that

$$
\begin{aligned}
\mathbb{E} \sup _{t \leq T} & \left|\int_{0}^{t} F(s) d Y(s)\right|^{p} \\
& \leq N\left[|R|_{1}^{p / 2}+\int_{K}|x|^{p} m(d x)+\left(\int_{K}|x|^{2} m(d x)\right)^{p / 2}\right] \mathbb{E} \int_{0}^{T}|F(s)|^{p} d s,
\end{aligned}
$$

where $R$ denotes the covariance operator of the Brownian component of $Y$.

Proof. Appealing to the Lévy-Itô's decomposition and Burkholder's inequality for stochastic integrals with respect to Hilbert space valued Wiener processes, we can assume, without loss of generality, that $R=0$. Let $k \in \mathbb{N}$ be such that $p \in\left[2^{k}, 2^{k+1}[\right.$. Then Burkholder's inequality (3.12) yields

$$
\mathbb{E}\left|\sup _{t \leq T} \int_{0}^{t} F(s) d Y(s)\right|^{p} \lesssim p \mathbb{E}\left(\int_{0}^{T}|F(s)|^{2} d[Y](s)\right)^{p / 2} .
$$

Setting

$$
\alpha=\mathbb{E}[Y](1)=\mathbb{E} \sum_{s \leq 1}|\Delta Y(s)|^{2}=\int_{K}|x|^{2} m(d x),
$$

we have that $\alpha<\infty$ and $[Y](t)-\alpha t$ is a martingale, because $[Y](t)$ is a onedimensional Lévy process. Moreover, we can write

$$
\begin{aligned}
\mathbb{E}\left(\int_{0}^{T}|F(s)|^{2}\right. & d[Y](s))^{p / 2} \\
& \left.\left.\lesssim_{p} \mathbb{E}\left|\int_{0}^{T}\right| F(s)\right|^{2} d([Y](s)-\alpha s)\right|^{p / 2}+\alpha^{p / 2} \mathbb{E}\left(\int_{0}^{T}|F(s)|^{2} d s\right)^{p / 2} .
\end{aligned}
$$

Applying again Burkholder's inequality to the first term on the right hand side of the previous expression one gets

$$
\left.\left.\mathbb{E}\left|\int_{0}^{T}\right| F(s)\right|^{2} d([Y](s)-\alpha s)\right|^{p / 2} \lesssim_{p} \mathbb{E}\left(\sum_{s \leq t}|F(s)|^{4}|\Delta Y(s)|^{4}\right)^{p / 4} .
$$

Since

$$
\left(\int_{0}^{T}|F(s)|^{2} d s\right)^{p / 2} \lesssim_{p, T} \int_{0}^{T}|F(s)|^{p} d s
$$

we obtain, iterating the above decomposition procedure,

$$
\begin{array}{rl}
\mathbb{E}\left|\sup _{t \leq T} \int_{0}^{t} F(s) d Y(s)\right|^{p} \lesssim_{p, T} & \mathbb{E}\left(\sum_{s \leq t}|F(s)|^{2^{k+1}}|\Delta Y(s)|^{2^{k+1}}\right)^{p / 2^{k+1}} \\
& +\left(\sum_{i=1}^{k}\left(\int|x|^{2^{i}} m(d x)\right)^{p / 2^{i}}\right) \mathbb{E} \int_{0}^{T}|F(s)|^{p} d s .
\end{array}
$$


We can write

$$
\begin{aligned}
\left(\sum_{s \leq t}|F(s)|^{2^{k+1}}|\Delta Y(s)|^{2^{k+1}}\right)^{\frac{p}{2^{k+1}}} & =\left(\sum_{s \leq t}\left(|F(s)|^{2^{k}}|\Delta Y(s)|^{2^{k}}\right)^{2}\right)^{\frac{1}{2} \frac{p}{2^{k}}} \\
& =:|a|_{\ell_{2}}^{p / 2^{k}} \leq|a|_{\ell_{p / 2^{k}}}^{p / 2^{k}}=\sum_{s \leq t}|F(s)|^{p}|\Delta Y(s)|^{p}
\end{aligned}
$$

where we have used the inequality $|a|_{\ell_{2}} \leq|a|_{\ell_{q}}$, which holds for all $a \in \ell_{q}, q \in[1,2]$. Since the process $|F(\cdot)|^{p}$ is predictable and $\sum_{s \leq t}|\Delta Y(s)|^{p}$ is an increasing adapted càdlàg process with compensator $t \mapsto t \int_{K}|x|^{p} m(d x)$, we have that

$$
\int_{0}^{t}|F(s)|^{p} d\left(\sum_{r \leq s}|\Delta Y(r)|^{p}-s \int_{K}|x|^{p} m(d x)\right)
$$

is a martingale with expectation zero. Therefore we can write

$$
\mathbb{E}\left|\int_{0}^{t} F(s) d Y(s)\right|^{p} \lesssim_{p, T}\left[\int_{K}|x|^{p} m(d x)+\sum_{i=1}^{k}\left(\int_{K}|x|^{2^{i}} m(d x)\right)^{p / 2^{i}}\right] \int_{0}^{t}|F(s)|^{p} d s
$$

In order to complete the proof it is then enough to show that

$$
\left(\int_{K}|x|^{2^{i}} m(d x)\right)^{p / 2^{i}} \leq\left(\int_{K}|x|^{2} m(d x)\right)^{p / 2}+\int_{K}|x|^{p} m(d x)
$$

for all $i \in[1, k]$. Define the probability measure on $K$

$$
\mu(d x)=\frac{1}{Z}|x|^{2} m(d x),
$$

where $Z=\int_{K}|x|^{2} m(d x)$, and set, for simplicity of notation, $q=2^{i}$. Then proving the above inequality is equivalent to proving that

$$
Z^{p / q}\left(\int_{K}|x|^{q-2} \mu(d x)\right)^{p / q} \leq Z^{p / 2}+Z \int_{K}|x|^{p-2} \mu(d x) .
$$

We distinguish two cases: if

$$
\left(\int_{K}|x|^{q-2} \mu(d x)\right)^{p / q} \leq Z^{p / 2-p / q}
$$

the inequality is obvious, otherwise, if

$$
Z \leq\left(\int_{K}|x|^{q-2} \mu(d x)\right)^{\frac{2}{q-2}}
$$

then it is sufficient to prove that

$$
Z^{p / q-1}\left(\int_{K}|x|^{q-2} \mu(d x)\right)^{p / q} \leq \int_{K}|x|^{p-2} \mu(d x),
$$

which follows immediately by the upper bound on $Z$ and Jensen's inequality. 
Remark 3.10. The fact that one can obtain a Bichteler-Jacod inequality with constant independent of the dimension is irrelevant for the purposes of [30], which deals with stochastic equations in $\mathbb{R}^{d}$, but appears to be known to specialists in Malliavin calculus for jumps - see e.g. lemma 5.1 and remark 5.2 in [4], and references therein.

For convenience we shall denote by $\mathfrak{m}_{p}$ the term in square parentheses appearing in (3.13). The same type of inequality can be established also for stochastic convolutions, even though in general they are not martingales.

Proposition 3.11. Let $A$ be the generator of a strongly continuous contraction semigroup on $E$ and $F:[0, T] \rightarrow \mathcal{L}_{2}(K, E)$ be a locally bounded predictable process. Let $p \in[2, \infty)$ and $M \in \mathcal{M}_{p}$. Then there exists a constant $N$, which depends only on $p$ and $T$, such that

$$
\mathbb{E} \sup _{t \leq T}\left|\int_{0}^{t} e^{(t-s) A} F(s) d M(s)\right|^{p} \leq N \mathfrak{m}_{p} \mathbb{E} \int_{0}^{T}|F(s)|^{p} d s .
$$

Proof. We shall follow the approach of [16]. In particular, by Sz.-Nagy's theorem on unitary dilations, there exists a Hilbert space $\bar{E}$, with $E$ isometrically embedded into $\bar{E}$, and a unitary strongly continuous group $T(t)$ on $\bar{E}$ such that $\pi T(t) x=e^{t A} x$ for all $x \in E, t \in \mathbb{R}$, where $\pi$ denotes the orthogonal projection from $\bar{E}$ to $E$. Then we have, recalling that the operator norms of $\pi$ and $T(t)$ are less or equal than one,

$$
\begin{aligned}
\mathbb{E} \sup _{t \leq T}\left|\int_{0}^{t} e^{(t-s) A} F(s) d M(s)\right|_{E}^{p} & =\mathbb{E} \sup _{t \leq T}\left|\pi T(t) \int_{0}^{t} T(-s) F(s) d M(s)\right|_{\bar{E}}^{p} \\
& \leq|\pi|^{p} \sup _{t \leq T}|T(t)|^{p} \mathbb{E}\left|\int_{0}^{t} T(-s) F(s) d M(s)\right|_{\bar{E}}^{p} \\
& \leq \mathbb{E} \sup _{t \leq T}\left|\int_{0}^{t} T(-s) F(s) d M(s)\right|_{\bar{E}}^{p} .
\end{aligned}
$$

Since the integral in the last expression is a martingale, inequality (3.13) implies that there exists a constant $N=N(p, T)$ such that

$$
\begin{aligned}
\mathbb{E} \sup _{t \leq T}\left|\int_{0}^{t} e^{(t-s) A} F(s) d M(s)\right|_{E}^{p} & \leq N \mathfrak{m}_{p} \mathbb{E} \int_{0}^{T}|T(-s) F(s)|^{p} d s \\
& \leq N \mathfrak{m}_{p} \mathbb{E} \int_{0}^{T}|F(s)|^{p} d s,
\end{aligned}
$$

where we used again that $T(t)$ is a unitary group and that the norms of $F(s)$ in $\mathcal{L}_{0}(K, \bar{E})$ and $\mathcal{L}_{0}(K, E)$ are equal.

We now have all the tools to prove theorem 3.7 .

Proof of theorem 3.7. As a first step we prove that the mapping $\mathfrak{F}: \mathbb{H}_{p}(T) \rightarrow \mathbb{H}_{p}(T)$ defined by

$$
\mathfrak{F} u(t)=e^{t A} u_{0}+\int_{0}^{t} e^{(t-s) A} f(s, u(s)) d s+\int_{0}^{t} e^{(t-s) A} B(s, u(s)) d M(s)
$$


is well defined and is a contraction. Let us prove that the image of $\mathfrak{F}$ is in fact contained in $\mathbb{H}_{p}(T)$. Predictability of $\mathfrak{F} u$ follows as in the proof of theorem 3.5, Let us prove that $\|\mathfrak{F} u\|_{p}<\infty$ : Minkowski's inequality yields

$$
\begin{array}{rl}
\|\mathfrak{F} u\|_{p}^{p} \lesssim p & \mathbb{E} \sup _{t \leq T}\left|e^{t A} u_{0}\right|^{p}+\mathbb{E} \sup _{t \leq T}\left|\int_{0}^{t} e^{(t-s) A} f(s, u(s)) d s\right|^{p} \\
+ & \mathbb{E} \sup _{t \leq T}\left|\int_{0}^{t} e^{(t-s) A} B(s, u(s)) d M(s)\right|^{p}
\end{array}
$$

where $\mathbb{E} \sup _{t \leq T}\left|e^{t A} u_{0}\right|^{p} \leq \sup _{t \leq T}\left|e^{t A}\right|^{p} \mathbb{E}\left|u_{0}\right|^{p}<\infty$. Moreover, by Cauchy-Schwarz' inequality and (3.4) we get

$$
\begin{aligned}
\mathbb{E} \sup _{t \leq T}\left|\int_{0}^{t} e^{(t-s) A} f(s, u(s)) d s\right|^{p} & \leq \mathbb{E} \sup _{t \leq T}\left[\int_{0}^{t}\left|e^{(t-s) A} f(s, u(s))\right| d s\right]^{p} \\
& \leq T^{p / 2} \mathbb{E} \sup _{t \leq T}\left[\int_{0}^{t}\left|e^{(t-s) A} f(s, u(s))\right|^{2} d s\right]^{p / 2} \\
& \leq T^{p / 2} \sup _{t \leq T}\left(\int_{0}^{t} h(t-s)^{2} d s\right)^{p / 2} \mathbb{E} \sup _{t \leq T}(1+|u(t)|)^{p}
\end{aligned}
$$

hence

$$
\mathbb{E} \sup _{t \leq T}\left|\int_{0}^{t} e^{(t-s) A} f(s, u(s)) d s\right|^{p} \lesssim\left(1+\mathbb{E} \sup _{t \leq T}|u(t)|^{p}\right)<\infty .
$$

Similarly, using the maximal inequality (3.14) and (3.5), we obtain

$$
\begin{aligned}
& \mathbb{E} \sup _{t \leq T}\left|\int_{0}^{t} e^{(t-s) A} B(s, u(s)) d M(s)\right|^{p} \lesssim_{p, T, m} \mathbb{E} \int_{0}^{T}|B(s, u(s))|^{p} d s \\
& \leq \mathbb{E} \int_{0}^{T} h(s)^{p}(1+|u(s)|)^{p} d s \\
& \lesssim\left(1+\mathbb{E} \sup _{s \leq T}|u(t)|^{p}\right) \int_{0}^{T} h(s)^{p} d s<\infty,
\end{aligned}
$$

thus completing the proof that $\mathfrak{F}\left(\mathbb{H}_{p}(T)\right) \subset \mathbb{H}_{p}(T)$.

Analogous calculations show that there exists a constant $N=N(T, h, p, m)$ such that $\|\mathfrak{F} u-\mathfrak{F} v\|_{p} \leq N\|u-v\|_{p}$, with $N\left(T_{0}, h, p, m\right)<1$ for some $T_{0}>0$. The existence and uniqueness proof is then finished exactly as in the proof of theorem 3.5. The estimate (3.10) is also established in the same way, with very minor modifications.

\section{Proofs of theorems 2.3 and 2.6}

In order to apply theorems 3.5 and 3.7 to (1.1) we need to prove that $B$ and $f$ satisfy Lipschitz conditions. Unfortunately we can only prove that $B$ and $f$ are locally Lipschitz, hence we obtain only a local existence and uniqueness result.

Let us briefly recall that the volatility coefficient $\sigma$ appearing in (1.1) is a random function from $[0, T] \times \mathbb{R}_{+} \times \mathbb{R}$ to $K$, and that the operator $B$ is defined as 
$[B(t, u) \phi](x)=\langle\sigma(t, x, u(x)), \phi\rangle_{K}$, for $\phi \in K$. Let us also define the space $\mathfrak{H}=$ $L_{2, \alpha}^{1}\left(\mathbb{R}_{+}, K\right)$, equipped with the norm

$$
|\phi|_{\mathfrak{H}}^{2}:=|\phi(0)|_{K}^{2}+\int_{0}^{\infty}\left|\phi^{\prime}(x)\right|_{K}^{2} \alpha(x) d x<\infty .
$$

Proposition 4.1. Assume that hypotheses (i)-(iv) of theorem 2.3 are satisfied. Then $B(t, u) \in \mathcal{L}_{2}(K, H)$ for all $u$ and there exists a constant $N$ such that $|B(t, u)|_{2} \leq$ $N(1+R)|u|$ and $|B(t, u)-B(t, v)|_{2} \leq N(1+R)|u-v|_{H}$ for all $u, v \in B_{R}(H)$, uniformly over $t \geq 0$.

Proof. We have

$$
|B(t, u)|_{2}^{2}=\sum_{k=1}^{\infty}\left|B(t, u) e_{k}\right|_{H}^{2}=\sum_{k=1}^{\infty} \int_{0}^{\infty}\left|\sigma^{k}(t, x, u(x))_{x}\right|^{2} \alpha(x) d x
$$

and

$$
\int_{0}^{\infty}\left|\sigma^{k}(t, x, u(x))_{x}\right|^{2} \alpha(x) d x \lesssim \int_{0}^{\infty}\left(\sigma_{x}^{k}(t, x, u(x))^{2}+\sigma_{u}^{k}(t, x, u(x))^{2} u^{\prime}(x)^{2}\right) \alpha(x) d x,
$$

where, using (2.9) and (2.4),

$$
\begin{aligned}
\int_{0}^{\infty}\left|\sigma_{x}^{k}(t, x, u(x))\right|^{2} \alpha(x) d x & \lesssim \int_{0}^{\infty}\left|\sigma_{x}^{k}(t, x, u(x))-\sigma_{x}^{k}(t, x, 0)\right|^{2} \alpha(x) d x+|\sigma(t, \cdot, 0)|_{\mathfrak{H}}^{2} \\
& \leq \int_{0}^{\infty} \beta^{k}(x)^{2} u(x)^{2} \alpha(x) d x+|\sigma(t, \cdot, 0)|_{\mathfrak{H}}^{2} \\
& \leq\left|\beta^{k}\right|_{\alpha}^{2}|u|_{\infty}^{2}+|\sigma(t, \cdot, 0)|_{\mathfrak{H}}^{2} \lesssim_{\alpha}\left|\beta^{k}\right|_{\alpha}^{2}|u|_{H}^{2}+|\sigma(t, \cdot, 0)|_{\mathfrak{H}}^{2},
\end{aligned}
$$

hence

$$
|B(t, u)|_{2}^{2} \leq|\beta|_{\alpha, \ell_{2}}^{2}|u|_{H}^{2}+|\sigma(t, \cdot, 0)|_{\mathfrak{H}}^{2}<\infty
$$

Similarly,

$$
|B(t, u)-B(t, v)|_{2}^{2}=\sum_{k=1}^{\infty} \int_{0}^{\infty}\left|\sigma^{k}(t, x, u(x))_{x}-\sigma^{k}(t, x, v(x))_{x}\right|^{2} \alpha(x) d x,
$$

and

$$
\begin{aligned}
\left|\left(\sigma^{k}(t, x, u(x))-\sigma^{k}(t, x, v(x))\right)_{x}\right|^{2} \lesssim & \left|\sigma_{x}^{k}(t, x, u(x))-\sigma_{x}^{k}(t, x, v(x))\right|^{2} \\
& +\left|\sigma_{u}^{k}(t, x, u(x)) u^{\prime}(x)-\sigma_{u}^{k}(t, x, v(x)) v^{\prime}(x)\right|^{2} .
\end{aligned}
$$

We also have

$$
\sum_{k=1}^{\infty} \int_{0}^{\infty}\left|\sigma_{x}^{k}(t, x, u(x))-\sigma_{x}^{k}(t, x, v(x))\right|^{2} \alpha(x) d x \leq|\beta|_{\alpha, \ell_{2}}^{2}|u-v|_{H}^{2},
$$


and, by virtue of (2.8) and (2.4),

$$
\begin{aligned}
& \sum_{k=1}^{\infty} \int_{0}^{\infty}\left|\sigma_{u}^{k}(t, x, u(x)) u^{\prime}(x)-\sigma_{u}^{k}(t, x, v(x)) v^{\prime}(x)\right|^{2} \alpha(x) d x \\
& \lesssim \quad \sum_{k=1}^{\infty} \int_{0}^{\infty}\left|\sigma_{u}^{k}(t, x, u(x)) u^{\prime}(x)-\sigma_{u}^{k}(t, x, u(x)) v^{\prime}(x)\right|^{2} \alpha(x) d x \\
& \quad+\sum_{k=1}^{\infty} \int_{0}^{\infty}\left|\sigma_{u}^{k}(t, x, u(x)) v^{\prime}(x)-\sigma_{u}^{k}(t, x, v(x)) v^{\prime}(x)\right|^{2} \alpha(x) d x \\
& \leq|\gamma|_{\ell_{2}}^{2}|u-v|_{1, \alpha}^{2}+|\gamma|_{\ell_{2}}^{2} \int_{0}^{\infty}\left|(u(x)-v(x)) v^{\prime}(x)\right|^{2} \alpha(x) d x \\
& \lesssim|u-v|_{H}^{2}+|u-v|_{\infty}^{2}|v|_{1, \alpha}^{2} \lesssim\left(1+|v|_{H}^{2}\right)|u-v|_{H}^{2},
\end{aligned}
$$

where in the second last step we have used the fact that $\left|\sigma_{u u}^{k}\right|<\gamma^{k}$ implies that $\sigma_{u}^{k}$ is Lipschitz with respect to $u$ with Lipschitz constant not greater than $\gamma^{k}$. The above estimates imply that $|B(t, u)-B(t, v)|_{2} \lesssim(1+R)|u-v|_{H}$ for all $|u|_{H},|v|_{H} \leq R$, hence also, by (4.1),

$$
|B(t, u)|_{2} \lesssim|B(t, 0)|_{2}+(1+R)|u| \leq|\sigma(t, \cdot, 0)|_{\mathfrak{H}}+(1+R)|u| \lesssim(1+R)|u| .
$$

We shall now obtain conditions under which the function $g$ defined in (2.3) satisfies a local Lipschitz condition. A useful tool is given by the following lemma, which essentially allows to obtain vector-valued versions of the inequalities (2.4)-(2.6).

Lemma 4.2. Let $\phi \in \mathfrak{H}$. Then $|\phi|_{K} \in H$ and $\left.\left.|| \phi\right|_{K}\right|_{H} \leq|\phi|_{\mathfrak{H}}$.

Proof. It is enough to prove the statement assuming $\phi(0)=0$, as it is immediate to see by comparing the definitions of $\|\left.\left.\cdot\right|_{K}\right|_{H}$ and $|\cdot|_{\mathfrak{H}}$. Let $\eta_{\varepsilon} \in C^{1}(K, \mathbb{R})$ be a smooth approximation of the norm of $K$, such that $\left|D \eta_{\varepsilon}(x)\right| \leq 1$ for all $x \in K$. Then we have

$$
\begin{aligned}
\left|\eta_{\varepsilon}(\phi)\right|_{H}^{2} & =\int_{0}^{\infty}\left|D_{x} \eta_{\varepsilon}(\phi(x))\right|_{K}^{2} \alpha(x) d x \\
& =\int_{0}^{\infty}\left|D \eta_{\varepsilon}(\phi(x))\right|_{K}^{2}\left|\phi^{\prime}(x)\right|^{2} \alpha(x) d x \\
& \leq \int_{0}^{\infty}\left|\phi^{\prime}(x)\right|_{K}^{2} \alpha(x) d x=|\phi|_{\mathfrak{H}}^{2}<\infty .
\end{aligned}
$$

Since the above bound does not depend on $\varepsilon$ and $\eta_{\varepsilon}(\phi(x)) \rightarrow|\phi(x)|_{K}$ as $\varepsilon \rightarrow 0$, we infer that $|\phi(x)|_{K} \in H$ and $\left.\left.|| \phi\right|_{K}\right|_{H} \leq|\phi|_{\mathfrak{H}}$.

Proposition 4.3. There exists a positive constant $N$ depending only on $\alpha$ and $\psi$ such that

$$
|g(\sigma)-g(\rho)|_{H} \leq N\left(1+R^{2}\right)|\sigma-\rho|_{\mathfrak{H}}
$$

for all $\sigma, \rho \in B_{R}(\mathfrak{H})$. 
Proof. By definition of $g$ one immediately gets

$$
|g(\sigma)-g(\rho)|_{H}=|g(\sigma(0))-g(\rho(0))|+|g(\sigma)-g(\rho)|_{1, \alpha}
$$

and $|g(\sigma(0))-g(\rho(0))| \lesssim_{\psi}|\sigma(0)-\rho(0)|_{K} \leq|\sigma-\rho|_{\mathfrak{H}}$. Setting $S(x)=-\int_{0}^{x} \sigma(y) d y$ and $R(x)=-\int_{0}^{x} \rho(y) d y$, a simple calculation reveals that

$$
\begin{aligned}
& \mid g(\sigma)-\left.g(\rho)\right|_{1, \alpha} ^{2} \\
& \lesssim \int_{0}^{\infty}\left|\left\langle\sigma^{\prime}(x), D \psi(S(x))\right\rangle-\left\langle\rho^{\prime}(x), D \psi(R(x))\right\rangle\right|^{2} \alpha(x) d x \\
& \quad+\int_{0}^{\infty}\left|\left\langle\sigma(x), D^{2} \psi(S(x)) \sigma(x)\right\rangle-\left\langle\rho(x), D^{2} \psi(R(x)) \rho(x)\right\rangle\right|^{2} \alpha(x) d x .
\end{aligned}
$$

Since $\psi \in C_{b}^{3}\left(B_{r}(K)\right)$, in particular $D \psi$ is Lipschitz and bounded on $B_{r}(K)$, we have

$$
\begin{aligned}
\left|\left\langle\sigma^{\prime}, D \psi(S)\right\rangle-\left\langle\rho^{\prime}, D \psi(R)\right\rangle\right|_{\alpha}^{2} \lesssim & \left|\left\langle\sigma^{\prime}, D \psi(S)\right\rangle_{K}-\left\langle\rho^{\prime}, D \psi(S)\right\rangle_{K}\right|_{\alpha}^{2} \\
& +\left|\left\langle\rho^{\prime}, D \psi(S)\right\rangle_{K}-\left\langle\rho^{\prime}, D \psi(R)\right\rangle_{K}\right|_{\alpha}^{2} \\
& { }_{\psi}|\sigma-\rho|_{\mathfrak{H}}^{2}+|\rho|_{\mathfrak{H}}^{2}\left(\int_{0}^{\infty}|\sigma(x)-\rho(x)|_{K} d x\right)^{2} \\
& \lesssim_{\alpha}|\sigma-\rho|_{\mathfrak{H}}^{2}+|\rho|_{\mathfrak{H}}^{2} \| \sigma-\left.\left.\rho\right|_{K}\right|_{H} ^{2} \\
\leq & \left(1+|\rho|_{\mathfrak{H}}^{2}\right)|\sigma-\rho|_{\mathfrak{H}}^{2}
\end{aligned}
$$

where the second last and last inequalities follow by (2.5) and lemma 4.2, respectively. Similarly we have

$$
\begin{aligned}
& \left|\left\langle\sigma, D^{2} \psi(S) \sigma\right\rangle-\left\langle\rho, D^{2} \psi(R) \rho\right\rangle\right|_{\alpha}^{2} \\
& \quad \lesssim\left|\left\langle\sigma, D^{2} \psi(S) \sigma\right\rangle-\left\langle\rho, D^{2} \psi(S) \rho\right\rangle\right|_{\alpha}^{2}+\left|\left\langle\rho,\left(D^{2} \psi(S)-D^{2} \psi(R)\right) \rho\right\rangle\right|_{\alpha}^{2} .
\end{aligned}
$$

Note that for any operator $Q \in \mathcal{L}(K)$ we have

$$
\begin{aligned}
|\langle Q x, x\rangle-\langle Q y, y\rangle|^{2} & \leq 2|\langle Q x, x\rangle-\langle Q x, y\rangle|^{2}+2|\langle Q x, y\rangle-\langle Q y, y\rangle|^{2} \\
& \leq 2|Q x|^{2}|x-y|^{2}+2|Q(x-y)|^{2}|y|^{2} \\
& \leq 2|Q|^{2}\left(|x|^{2}+|y|^{2}\right)|x-y|^{2}
\end{aligned}
$$

therefore, by Cauchy-Schwarz' inequality, (2.6), and lemma 4.2 .

$$
\begin{aligned}
& \left|\left\langle\sigma, D^{2} \psi(S) \sigma\right\rangle-\left\langle\rho, D^{2} \psi(S) \rho\right\rangle\right|_{\alpha}^{2} \\
& \lesssim_{\psi} \int_{0}^{\infty}\left(|\sigma(x)|_{K}^{2}+|\rho(x)|_{K}^{2}\right)|\sigma(x)-\rho(x)|_{K}^{2} \alpha(x) d x \\
& \left.\lesssim\left(\int_{0}^{\infty}\left(|\sigma(x)|_{K}^{4}+|\rho(x)|_{K}^{4}\right) \alpha(x) d x\right)^{1 / 2}\left(\int_{0}^{\infty}|\sigma(x)-\rho(x)|_{K}^{4}\right) \alpha(x) d x\right)^{1 / 2} \\
& \lesssim_{\alpha}\left(\left.\left.|| \sigma\right|_{K}\right|_{H} ^{2}+\left.\left.|| \rho\right|_{K}\right|_{H} ^{2}\right)|| \sigma-\left.\left.\rho\right|_{K}\right|_{H} ^{2} \leq\left(|\sigma|_{\mathfrak{H}}^{2}+|\sigma|_{\mathfrak{H}}^{2}\right)|\sigma-\rho|_{\mathfrak{H}}^{2} .
\end{aligned}
$$

Finally, since $D^{2} \psi$ is Lipschitz, applying (2.5), (2.6) and lemma 4.2, we get

$$
\begin{aligned}
\left|\left\langle\rho,\left(D^{2} \psi(S)-D^{2} \psi(R)\right) \rho\right\rangle\right|_{\alpha}^{2} & \left.\left.\lesssim_{\psi}|| \rho\right|_{K} ^{2}\right|_{\alpha} ^{2}\left(\int_{0}^{\infty}|\sigma(x)-\rho(x)|_{K} d x\right)^{2} \\
& \lesssim_{\alpha}|\rho|_{\mathfrak{H}}^{4}|| \sigma-\left.\left.\rho\right|_{K}\right|_{H} ^{2} \leq|\rho|_{\mathfrak{H}}^{4}|\sigma-\rho|_{\mathfrak{H}}^{2},
\end{aligned}
$$

from which the claim follows. 
Proof of theorem 2.3. Let $u, v \in B_{R}(H)$. Then we have $|\sigma(t, u)-\sigma(t, v)|_{\mathfrak{H}} \leq N(1+$ $R)|u-v|_{H}$. In fact, obvious steps show that

$$
\begin{aligned}
|\sigma(t, u)-\sigma(t, v)|_{\mathfrak{H}}^{2} & =\int_{0}^{\infty}\left|\sigma(t, x, u(x))_{x}-\sigma(t, x, v(x))_{x}\right|_{K}^{2} \alpha(x) d x \\
& \leq|B(t, u)-B(t, v)|_{2}^{2} \leq N(1+R)^{2}|u-v|_{H}^{2},
\end{aligned}
$$

where the last inequality follows by proposition 4.1. This estimate, together with proposition 4.3, implies that there exists a constant $N$ such that $|f(t, u)-f(t, v)|_{H} \leq$ $N\left(1+R^{2}\right)|u-v|_{H}$ for all $u, v \in B_{R}(H)$. In turns, this implies that $f$ is locally bounded, as a consequence of hypothesis (iii). Similarly, $B$ is locally bounded, as follows by (4.1). The proof is then finished in a standard way appealing to theorem 3.5

Proof of theorem 2.6. Let us denote by $\dot{H}$ the space $L_{2, \alpha}^{1}$ endowed with the norm

$$
|\phi|_{*}^{2}=\phi(\infty)^{2}+\int_{0}^{\infty} \phi^{\prime}(x)^{2} \alpha(x) d x
$$

which is equivalent to the norm of $H$. Moreover, define as $\dot{H}_{0}$ the subspace of functions $\phi \in \dot{H}$ such that $\phi(\infty)=0$. Then the semigroup $e^{t A}$ is a contraction in $\dot{H}_{0}$, because

$$
\left|e^{t A} \phi\right|_{*}^{2}=\int_{t}^{\infty} \phi^{\prime}(x)^{2} \alpha(x) d x \leq|\phi|_{H}^{2} .
$$

Therefore we can apply theorem 3.7, noting that the necessary properties of $f$ and $B$ are proved as in the previous proof. We thus obtain the existence and uniqueness of a solution $u$ with values in $\dot{H}$. But since the norms of $H$ and $\dot{H}$ are equivalent, $u$ is also well defined as a process in $\mathbb{H}_{p}(T)$.

Remark 4.4. Note that instead of starting from equation (1.1), one could start directly from an abstract evolution equation like (2.7), with $B$ an operator depending on the whole forward curve $u(t, \cdot)$. In fact, roughly speaking, (1.1) is just a special case of (2.7) where $B$ is the Nemitski operator associated to $\sigma$. Unfortunately we have only been able to prove that such $B$ is locally Lipschitz with respect to $u$. Nothing forbids to assume that, in the general case, $B$ is Lipschitz, but even so we could only prove that $f$ is locally Lipschitz, hence mild solutions would still be only local.

\section{$5 \quad$ Examples}

\subsection{Finite dimensional noise}

Let us consider, for simplicity, the case $K=\mathbb{R}$ (the more general case $K=\mathbb{R}^{d}$, $d<\infty$, being similar). Let $M$ be a real valued Lévy process such that $M(1)$ admits an analytic characteristic function, e.g. a jump-diffusion (i.e. the sum of a Wiener process and a Poisson process) or a Gamma process (which are discussed, for instance, in [1]). Then, thanks to the analyticity of the characteristic function of $M(1)$, we infer that $\psi \in C^{\infty}\left(B_{r}\right)$ (see e.g. [23]). In particular, since $B_{r}$ is compact, $\psi \in C_{b}^{3}\left(B_{r}\right)$, and all the hypotheses on $M$ of section 2 are satisfied. 


\subsection{Infinitely many independent noise sources}

Consider a model of the type (1.1), where $\sigma=\sum_{k} \sigma^{k} e_{k}$ and $M$ is formally defined as

$$
M(t)=\sum_{k=1}^{\infty} \xi^{k}(t) e_{k}
$$

where $\xi^{k}, k \in \mathbb{N}$, are real independent Lévy processes. In order to consider the problem in the setting developed above, we need to establish conditions under which $M$ is a well defined Lévy process on the Hilbert space $K$.

Lemma 5.1. Let $\xi^{k}, k \in \mathbb{N}$, be real independent Lévy processes with characteristic triplets $\left(b^{k}, r^{k}, m^{k}\right), k \in \mathbb{N}$. If $b, r \in \ell_{2}$ and

$$
\sum_{k=1}^{\infty} \int_{\mathbb{R}}\left(1 \wedge x^{2}\right) m^{k}(d x)<\infty,
$$

then (5.1) defines a Lévy process on $K$ with characteristic triplet $(b, R, m)$, where $b=\sum_{k=1}^{\infty} b^{k} e_{k}, R: K \ni y \mapsto \sum r^{k}\left\langle y, e_{k}\right\rangle e_{k}$, and $m: \mathcal{B}(K) \ni A \mapsto \sum m^{k}\left(A^{k}\right)$, where $A^{k}$ denotes the projection of $A$ on $\operatorname{span}\left(e_{k}\right)$.

Proof. One has, using the summation convention over repeated indices (when no confusion arises),

$$
\mathbb{E} e^{i\langle a, M(t)\rangle}=\mathbb{E} e^{i\left\langle a^{k} e_{k}, \xi_{t}^{k} e_{k}\right\rangle}=\mathbb{E} e^{i a_{k} \xi_{t}^{k}}=e^{-t \sum \lambda^{k}\left(a^{k}\right)},
$$

and

$$
\begin{aligned}
\lambda(a) & :=\left(\sum \lambda^{k}\right)\left(a^{k}\right)=-i b^{k} a^{k}+\frac{1}{2} r^{k}\left(a^{k}\right)^{2}-\sum \int_{\mathbb{R}}\left(e^{i a^{k} x}-1-i \frac{a^{k} x}{1+x^{2}}\right) m^{k}(d x) \\
& =-i\langle b, a\rangle+\frac{1}{2}\langle R a, a\rangle-\int_{H}\left(e^{i\langle a, x\rangle}-1-i \frac{\langle a, x\rangle}{1+|x|^{2}}\right) m(d x),
\end{aligned}
$$

i.e. $\mathbb{E} e^{i\langle a, M(t)\rangle}=e^{-t \lambda(a)}$, which is equivalent to the claim of the lemma.

Let us now consider the properties of the function $\psi$. We have

$$
\begin{aligned}
\psi(z) & =\log \int_{K} e^{\langle x, z\rangle} \mu(d x)=\log \prod_{k=1}^{\infty} \int_{\mathbb{R}} e^{x z^{k}} \mu^{k}(d x) \\
& =\sum_{k=1}^{\infty} \log \int_{\mathbb{R}} e^{x z^{k}} \mu^{k}(d x),
\end{aligned}
$$

where $\mu$ is the law of $M(1)$ and $\mu^{k}$ is the law of $\xi^{k}(1)$, for all $k \in \mathbb{N}$. Let us define the function $\varphi: B_{\delta r}\left(\ell_{2}\right) \rightarrow \mathbb{R}^{\infty}$ as

$$
\varphi: z^{\cdot} \mapsto \log \int_{\mathbb{R}} e^{x z^{k}} \mu^{k}(d x) .
$$


If the measures $\mu^{k}$ are such that the image of $B_{\delta r}\left(\ell_{2}\right)$ under $\varphi$ is contained in $\ell_{1}$, then (2.1) is satisfied. In particular, this is true if

$$
k \mapsto \log \int_{\mathbb{R} \backslash[-1,1]} e^{\left|z^{k}\right||x|} m^{k}(d x) \in \ell_{1}
$$

for all $z^{\cdot} \in B_{\delta r}\left(\ell_{2}\right)$, as it follows by theorem 25.3 of [32]. Consider for instance infinitely many independent Gamma processes $\left(\xi^{k}\right)_{k \in \mathbb{N}}$. Then the Lévy measure of $\mu^{k}$ is given by

$$
m^{k}(d x)=c^{k} x^{-1} e^{-\alpha^{k} x} d x, \quad x>0,
$$

where $c^{k}, \alpha^{k}$ are positive numbers. In the Lévy-Kintchine representation of $\mu^{k}$ one has $r^{k}=0$ and $b^{k}=c^{k}\left(\alpha^{k}\right)^{-1}$. Therefore

$$
\sum_{k=1}^{\infty} \int_{0}^{\infty}\left(1 \wedge x^{2}\right) m^{k}(d x) \leq \sum_{k=1}^{\infty} \int_{0}^{\infty} x^{2} m^{k}(d x),
$$

and the last integral is finite if

$$
\sum_{k=1}^{\infty} \int_{0}^{\infty} x^{2} \mu^{k}(d x)=\sum_{k=1}^{\infty}\left(\alpha^{k}\right)^{-2}\left(c^{k}+\left(c^{k}\right)^{2}\right) .
$$

Therefore, assuming that $\left(\alpha^{k}\right)^{-1} \in \ell_{\infty}$ and $c^{k} \in \ell_{1} \cap \ell_{2}$, lemma 5.1 implies that the series $\sum_{k} \xi^{k}(t) e_{k}$ defines a Lévy process on the Hilbert space $K$. We also have

$$
\log \int_{\mathbb{R}} e^{x z^{k}} \mu_{k}(d x)=-c^{k} \log \left(1-\left(\alpha^{k}\right)^{-1} z_{k}\right), \quad z^{k}<\alpha^{k},
$$

hence the $\ell_{1}$ norm of the left-hand side is bounded from above by

$$
|c|_{\ell_{1}}\left|\log \left(1-|1 / \alpha|_{\ell_{\infty}} \delta r\right)\right|,
$$

where $1 / \alpha$ stands for the sequence $\left(1 / \alpha^{k}\right)_{k \in \mathbb{N}}$. This implies that choosing $\delta$ and $r$ so that $\delta r<|1 / \alpha|_{\ell_{\infty}}^{-1}$, we obtain the local well-posedness of an HJM model driven by infinitely many Gamma processes, as follows by the results of section 2 .

\section{Conclusions}

We have proved existence and uniqueness of local mild solutions to Musiela's SPDE in a Hilbert space $H$ of absolutely continuous functions, that seems to be the standard "ambient space" for forward curves. While this space has several nice features, which are extensively discussed in [11, its main drawback is probably that it does not allow one to characterize forward curves at a given time $T>0$ as solutions of an SDE in $H$ (or at least we failed to find sufficient conditions). It is known that if $M$ is a Wiener process, then a unique global solution exists in weighted $L_{2}$ spaces (see e.g. [13]). On the other hand, such spaces are too big, in the sense that very rough (with respect to $x$ ) forward curves are allowed, and this should be ruled out in any sensible model for the dynamics of forward rates (see also the discussion in 
[10]). Therefore, it seems that one should find the "right" space to study Musiela's SPDE, satisfying the minimum requirement that its elements admit a continuous modification, and allowing at the same time to obtain existence and uniqueness of global mild solutions. This problem, to the best of our knowledge, is not solved also in the case of Brownian noise.

\section{Acknowledgments}

This work was partially supported by the DFG through the SFB 611, Bonn, and by the ESF through grant AMaMeF 969. This work was carried out while the author was visiting the Max-Planck-Institut für Mathematik in Leipzig supported by an EPDI fellowship. The author is sincerely grateful to S. Albeverio for helpful discussions on some parts of the paper, to two anonymous referees and to E. Eberlein for suggestions which led to improvements of the presentation.

\section{References}

[1] S. Albeverio, E. Lytvynov, and A. Mahnig, A model of the term structure of interest rates based on Lévy fields, Stochastic Process. Appl. 114 (2004), no. 2, 251-263. MR MR2101243 (2005h:60178)

[2] S. Albeverio, V. Mandrekar, and B. Rüdiger, Existence of mild solutions for stochastic differential equations and semilinear equations with non-Gaussian Lévy noise, Preprint SFB 611, Bonn, 2006.

[3] A. Ambrosetti and G. Prodi, A primer of nonlinear analysis, Cambridge University Press, Cambridge, 1995. MR MR1336591 (96a:58019)

[4] K. Bichteler, J.-B. Gravereaux, and J. Jacod, Malliavin calculus for processes with jumps, Gordon and Breach Science Publishers, New York, 1987. MR MR1008471 (90h:60056)

[5] T. Björk, G. Di Masi, Yu. Kabanov, and W. Runggaldier, Towards a general theory of bond markets, Finance Stochast. 1 (1997), 141-174.

[6] G. Da Prato and J. Zabczyk, Stochastic equations in infinite dimensions, Cambridge UP, 1992.

[7] E. Eberlein, J. Jacod, and S. Raible, Lévy term structure models: no-arbitrage and completeness, Finance Stoch. 9 (2005), no. 1, 67-88. MR MR2210928

[8] E. Eberlein and F. Özkan, The defaultable Lévy term structure: ratings and restructuring, Math. Finance 13 (2003), no. 2, 277-300. MR MR1967777 (2004a:91065)

[9] E. Eberlein and S. Raible, Term structure models driven by general Lévy processes, Math. Finance 9 (1999), no. 1, 31-53. MR MR1849355 (2002f:91039)

[10] I. Ekeland and E. Taflin, A theory of bond portfolios, Ann. Appl. Probab. 15 (2005), no. 2, 1260-1305. MR MR2134104 (2006b:91067)

[11] D. Filipović, Consistency problems for Heath-Jarrow-Morton interest rate models, Lecture Notes in Mathematics, vol. 1760, Springer-Verlag, Berlin, 2001. MR MR1828523 (2002e:91001) 
[12] D. Filipović and S. Tappe, Existence of Lévy term structure models, preprint. To appear in Finance Stoch., 2007.

[13] B. Goldys and M. Musiela, Infinite dimensional diffusions, Kolmogorov equations and interest rate models, Option pricing, interest rates and risk management, Handb. Math. Finance, Cambridge Univ. Press, Cambridge, 2001, pp. 314-335. MR 1848556

[14] I. Gyöngy, On stochastic equations with respect to semimartingales. III, Stochastics 7 (1982), no. 4, 231-254.

[15] H. D. Hamedani and B. Z. Zangeneh, Stopped Doob inequality for p-th moment, $0<p<\infty$, stochastic convolution integrals, Stochastic Anal. Appl. 19 (2001), no. 5, 771-798. MR MR1857896 (2002h:60120)

[16] E. Hausenblas and J. Seidler, A note on maximal inequality for stochastic convolutions, Czechoslovak Math. J. 51(126) (2001), no. 4, 785-790. MR MR1864042 (2002j:60092)

[17] A. Ichikawa, Some inequalities for martingales and stochastic convolutions, Stochastic Anal. Appl. 4 (1986), no. 3, 329-339. MR MR857085 (87m:60105)

[18] J. Jakubowski and J. Zabczyk, Exponential moments for HJM models with jumps, Finance Stoch. 11 (2007), no. 3, 429-445. MR MR2322920

[19] C. Knoche, SPDEs in infinite dimension with Poisson noise, C. R. Math. Acad. Sci. Paris 339 (2004), no. 9, 647-652. MR MR2103204

[20] P. Kotelenez, A submartingale type inequality with applications to stochastic evolution equations, Stochastics 8 (1982/83), no. 2, 139-151.

[21] _ A stopped Doob inequality for stochastic convolution integrals and stochastic evolution equations, Stochastic Anal. Appl. 2 (1984), no. 3, 245-265.

[22] P. Lescot and M. Röckner, Perturbations of generalized Mehler semigroups and applications to stochastic heat equations with Lévy noise and singular drift, Potential Anal. 20 (2004), no. 4, 317-344.

[23] E. Lukacs, Characteristic functions, Hafner Publishing Co., New York, 1970. MR MR0346874 (49 \#11595)

[24] C. Marinelli, Well-posedness and invariant measures for HJM models with deterministic volatility and Lévy noise, preprint arXiv:math.PR/0702622, 2007.

[25] M. Métivier, Semimartingales, Walter de Gruyter \& Co., Berlin, 1982. MR MR688144 (84i:60002)

[26] M. Métivier and G. Pistone, Une formule d'isométrie pour l'intégrale stochastique hilbertienne et équations d'évolution linéaires stochastiques, Z. Wahrscheinlichkeitstheorie und Verw. Gebiete 33 (1975/76), no. 1, 1-18. MR MR0383527 (52 \#4408)

[27] _ Sur une équation d'évolution stochastique, Bull. Soc. Math. France 104 (1976), no. 1, 65-85. MR MR0420854 (54 \#8866)

[28] Sz. Peszat and J. Zabczyk, Stochastic heat and wave equations driven by an impulsive noise, Stochastic partial differential equations and applications-VII, 
Lect. Notes Pure Appl. Math., vol. 245, Chapman \& Hall/CRC, Boca Raton, FL, 2006, pp. 229-242. MR MR2227232

[29] — Heath-Jarrow-Morton-Musiela equation of bond market, IMPAN preprint, 2007.

[30] Ph. Protter and D. Talay, The Euler scheme for Lévy driven stochastic differential equations, Ann. Probab. 25 (1997), no. 1, 393-423. MR MR1428514 (98c:60063)

[31] Ph. E. Protter, Stochastic integration and differential equations, second ed., Springer-Verlag, Berlin, 2004. MR MR2020294 (2005k:60008)

[32] Ken-iti Sato, Lévy processes and infinitely divisible distributions, Cambridge University Press, Cambridge, 1999. MR MR1739520 (2003b:60064)

[33] M. Tehranchi, A note on invariant measures for HJM models, Finance Stoch. 9 (2005), no. 3, 389-398. MR MR2211714

[34] T. Vargiolu, Invariant measures for the Musiela equation with deterministic diffusion term, Finance Stoch. 3 (1999), no. 4, 483-492. MR 2002i:60080 